\title{
Dilemma of the differential diagnosis of hilar cholangiocarcinoma and benign diseases:a single-center retrospective study
}

Liwei Pang ( $\sim 546790762 @ q q . c o m$ )

shengjing Shenyang

Research article

Keywords: Hilar cholangiocarcinoma, Hilar benign diseases, Differential diagnosis, Laboratory examination

Posted Date: July 6th, 2019

DOI: https://doi.org/10.21203/rs.2.11063/v1

License: (1) This work is licensed under a Creative Commons Attribution 4.0 International License. Read Full License

Version of Record: A version of this preprint was published at Carcinogenesis on November 18th, 2020. See the published version at https://doi.org/10.1093/carcin/bgaa125. 


\section{Abstract}

Objective Hilar cholangiocarcinoma, which lacks specific clinical manifestations, remains very difficult to distinguish from benign disease. This distinction is further complicated by the complex hilar anatomy. We conducted the present study to evaluate the differential diagnosis of these conditions. Methods Sixty-five patients underwent resection surgery for suspected hilar cholangiocarcinoma between January 2011 and October 2018. Following a postoperative pathology analysis, all patients were divided into 2 groups: malignant group (54 patients with HCCA) and benign group (11 cases with benign lesions). The patients' clinical data, including general demographics (sex, age), clinical manifestations (jaundice, abdominal discomfort, fever, weight loss), laboratory data [alanine aminotransferase (ALT), aspartate aminotransferase (AST), alkaline phosphatase (ALKP), total bilirubin (BILT), indirect bilirubin (BILD), carbohydrate antigen (CA) 19-9, carcinoembryonic antigen (CEA)], and imaging findings, were included in a retrospective analysis. Results Compared to the benign group, the malignant group had a significantly higher median age and serum CA19-9, CEA, ALT, BILT, and BILD levels $(P<0.05)$. By contrast, the groups did not differ significantly in terms of the sex distribution, clinical manifestations, serum levels of AST and ALKP, and imaging findings. In a receiver operating characteristic curve analysis, we identified a CA19-1 cut-off point of $233.15 \mathrm{U} / \mathrm{ml}$ for the differential diagnosis, with a sensitivity of $56 \%$ and specificity of $91 \%$. Furthermore, we identified a CEA cut-off point of $2.98 \mathrm{ng} / \mathrm{ml}$ for the differential diagnosis, with a sensitivity of $61 \%$ and specificity of $90 \%$. Conclusion The differential diagnosis of HCCA and benign hilar lesions remains difficult. However, we found that patients with HCCA tended to have an older age at onset and higher serum levels of CA19-9, CEA, BILT, ALT, and BILD. Furthermore, patients with a serum CA19-9 level $>233.15 \mathrm{U} / \mathrm{ml}$ and CEA level $>2.98 \mathrm{ng} / \mathrm{ml}$ are $\mathrm{more}$ likely to have malignant disease.

\section{Background}

Although many conditions can lead to hilar bile duct stenosis, most cases are attributable to hilar cholangiocarcinoma (HCCA) and benign inflammation[1]. HCCA, also known as Klatskin tumor, is a rare but devastating malignant disease with a poor long-term prognosis. This tumor type originates from the epithelial cells of the extrahepatic bile duct and accounts for $60-70 \%$ of all bile duct carcinomas[2,3]. The known risk factors for HCCA include chronic trematode infection, primary sclerosing cholangitis (PSC), abnormal biliary tract development, intrahepatic bile duct stones, and hepatitis B and/or hepatitis C infection[4].

Notably, many benign hilar lesions can mimic malignancy. Particularly, inflammatory lesions such as (PSC) and IgG4-associated sclerosing cholangitis (IAC) may present with the same clinical and radiological features as HCCA, including thickening of the bile duct wall, stenosis, and intrahepatic bile duct dilatation[12]. Several studies[5-8] have noted that approximately $14 \%$ to $25 \%$ of patients resected for presumed HCCA prove to have a benign lesion at histopathology.In order to avoid meaningless aggressive surgical treatment, differential diagnosis of benign and malignant tumors should be made before the operation.Currently, surgery remains the best curative option for highly suspected HCCA. The standard option involves bile duct resection accompanied with hepatectomy, caudate lobectomy, lymphadenectomy, and even vascular resection and reconstruction[32,33]. Many prognostic factors, including the lymph node status, tumor differentiation, and margin status have been reported to influence survival outcomes,[9-11] and the 5-year survival rates of $10-40 \%$ are far from satisfactory[34-36]. The surgical options for benign lesions differ somewhat, and include Roux-en$\mathrm{Y}$, hemi hepatectomy, common bile duct exploration, and T tube drainage. However, several histopathologic studies[5-8] have noted that $14-25 \%$ of resected lesions from patients with suspected HCCA are proven to be benign.

Given the above factors, the clinical features, diagnosis, and treatment of HCCA and benign diseases remains an enormous challenge, and the implementation of unnecessary surgery is an even greater cause for concern. Therefore, the differential diagnosis of benign and malignant tumors should be made preoperatively to avoid unnecessary aggressive surgeries. The present study aimed to investigate the differential diagnosis of HCCA and benign hilar diseases by comparing the imaging and diagnostic features of both lesion types and to determine the appropriate laboratory features that could potentially distinguish malignant lesions from benign biliary strictures.

\section{Methods}

\section{General information}

Sixty-five patients underwent resection surgery for suspected HCCA between January 2011 and October 2018. Following a postoperative pathologic analysis, the patients were divided into 2 groups: the malignant group comprised 54 cases with HCCA lesions and the benign group comprised 11 cases with benign lesions.All the patients had signed patient consent. The study was submitted to and approved by our institutional ethics committee. 


\section{Data extraction}

From all patients, the following clinical data were collected and analyzed for potentially differentiating factors: general demographics (sex, age), clinical manifestations (jaundice, abdominal discomfort, fever, weight loss), laboratory examination [levels of alanine aminotransferase (ALT), aspartate aminotransferase (AST), alkaline phosphatase (ALKP), total bilirubin (BILT), indirect bilirubin (BILD), carbohydrate antigen (CA) 19-9, and carcinoembryonic antigen (CEA)], imaging examination [3-dimensional (3D) color Doppler ultrasound, computed tomography (CT), magnetic resonance (MR)+MR cholangiopancreatography (MRCP)].

\section{Statistical analysis}

Data are expressed as means \pm standard deviations and were analyzed using SPSS, version 23.0 (IBM, Inc., Armonk, NY, USA). The measurement data are presented as medians (M) and quartiles (Q25, Q75), and numerical data are expressed as frequencies. Student's $t$ test was used to evaluate independent samples. A P value $\leq 0.05$ was considered statistically significant. The sensitivity, specificity, and area under the receiver operating characteristic (ROC) curve (AUC) were also calculated.

\section{Results}

1. Pathology and operation of patients (Table 1)

Table 1. Pathology and operation of patients

2. Clinical findings of patients with malignant and benign disease

2.1 Sex, age, and time from the appearance of symptoms to treatment

The malignant group included 31 men and 23 women. Three patients had a history of hepatitis B and 2 had a history of cholecystectomy. The benign group included 3 men and 8 women. Two had a history of previous cholecystectomy and 1 had a history of gallbladder-protected lithotomy. Although the groups did not differ significantly in terms of the sex distribution and disease process $(P$ $>0.05)$, the malignant group had a significantly higher median age $(P<0.01)$.

\subsection{Clinical features}

Both groups had fairly high incidence rates of jaundice and abdominal discomfort (>45\%), with no significant differences between the groups $(P>0.05)$. Furthermore, both groups had relatively lower incidence rates of fever and weight loss $(<27 \%)$, with no significant intergroup differences $(P>0.05)$.

\subsection{Serological examination}

Notably, the malignant group had significantly higher serum levels of CA19-9, CEA, ALT, BILT, and BILD, compared to the benign group (P $<0.05)$. However, the groups did not differ significantly in terms of the serum levels of AST and ALKP $(P>0.05)$.

Table 2. Comparison of the demographic, clinical, and laboratory characteristics between patients with hilar cholangiocarcinoma and those with benign bile strictures.

\section{Imaging examination}

In the malignant group, 41, 51, and 42 patients underwent 3D color Doppler ultrasound, CT, and MRCP, respectively. In the benign group, 10,11 , and 9 patients underwent these imaging scans, respectively. However, a comparison of the incidence of intrahepatic bile duct dilatation, bile duct wall thickening, vascular invasion, lymph node enlargement, and gallbladder invasion did not reveal significant differences between the benign and malignant groups $(P>0.05)$.

Table 3. Imaging findings in the benign and malignant groups

3. ROC curve analysis of serum CEA and CA19-9 levels

Next, we generated ROC curves of the serum levels of CA19-9 and CEA (Figures 1, 2), which yielded AUCs of 0.801 and 0.774 , respectively (both $\mathrm{P}<0.05$ ). For each factor, we determined a boundary or cut-off value corresponding to the maximum sum of sensitivity and specificity and calculated the boundary and related evaluation data. Furthermore, the ROC curve analyses of CEA and CA19-9 were 
performed to explicitly define the relationship of these factors with HCCA (Figures 1, 2 and Table 4). For CA19-9, a diagnostic boundary for differential diagnosis of $233.15 \mathrm{U} / \mathrm{ml}$ yielded a sensitivity and specificity of $56 \%$ and $91 \%$, respectively. Meanwhile, for CEA, a diagnostic boundary of $2.98 \mathrm{mg} / \mathrm{ml}$ yielded a sensitivity and specificity of $61 \%$ and $90 \%$, respectively.

Table 4. Relevant evaluation data determined from the boundary points

\section{Discussion}

Hilar bile duct stenosis is unusual and the most common causes include hilar cholangiocarcinoma (HCCA) and inflammatory lesions.Surgical resection is the only curative treatment option for patients with HCCA and many prognostic factors such as lymph node status, tumor differentiation, margin status have been reported to influence survival outcomes[9-11].However,some benign disease such as primary sclerosing cholangitis(PSC) and IgG4-associated sclerosing cholangitis(IAC) may resemble those of HCCA, including thickening of the bile duct wall, stenosis and intrahepatic bile duct dilatation[12]. Undertaking unnecessary surgery for suspicious of hilar cholangiocarcinoma becomes even more debate when many cases of misdiagnosis have been reported.But the clinical features, diagnosis, treatment a in HCCA with benign diseases still is an enormous challenge.

In this study, we found no differences between the malignant and benign groups in terms of sex, body mass index, or clinical symptoms (Table 2). Furthermore, patients in both groups presented with similar clinical symptoms, which made it very difficult to distinguish these two types of diseases. These findings are consistent with a lack of unique clinical manifestations during the early stage of HCCA. Consequently, most affected patients are only treated upon the manifestation of severe biliary obstruction or jaundice as demonstrated by Paritpokee[13], who believed that painless progressive jaundice combined with anorexia was highly suspicious of malignancy.

In we observed a statistically significant difference in age between the groups, as well as an indication of a poorer prognosis for HCCA. Furthermore, patients with HCCA had significantly higher serum ALT and BILT levels (Table 2). These increases in serum BILT and ALP levels are consistent with previous studies and may be attributable to the release of enzymes from tumor cells, tumor infiltration and growth along the bile duct wall, a wider and/or more severe stenosis, and greater damage to liver function[14,15]. Still, these findings are not very useful predictors of malignancy.

However, patients with HCCA in our study also had significantly higher serum levels of CA19-9 and CEA (Table 2). However, an elevated level of CA19-9 or CEA in isolation cannot be used to accurately distinguish benign and malignant diseases[16]. One previous study reported that a combination of CA $19-9$ and CEA yielded a diagnostic sensitivity of $100 \%$ sensitivity[17], but these results were not confirmed by other researchers[12,18]. More recent studies have identified the IgG4 as a potential serum marker for differentiating benign disease from HCCA, although further investigation is needed to clarify the diagnostic value and the role of immunomodulatory drugs $[12,19,20]$. Other recent studies have evaluated a more promising tumor marker, human mucin 5 subtypes A and C (MUC5AC), which yielded a diagnostic sensitivity and specificity of $71 \%$ and $90 \%$, respectively[21]. Still, painless jaundice, together with increased serum levels of CA 19-9 and CEA, tends to associate with HCCA rather than with benign disease.

Imaging techniques have improved during the last decade, and current diagnostic work-ups of patients with suspected HCCA usually comprise ultrasound, enhanced CT, and MRCP. On ultrasound, cholangiocarcinoma typically presents the following features[22,23]: (1) obvious dilatation of the intrahepatic bile duct, truncation of the hepatic hilum, and mass echo; (2) empty gallbladder, non-dilatation of the extrahepatic bile duct, and gallbladder swelling when cancer invades the gallbladder duct; and (3) no enlargement of the pancreatic head. Although abdominal ultrasound is a noninvasive, repeatable, simple, and economical first-line option for the clinical determination of biliary tract dilatation, stone removal, and degree of obstruction, it cannot be used to accurately determine the form of obstruction or the extent of tumor invasion. Ultrasound is also dependent on the experience of the examiner and interference from gastrointestinal gas, among other factors[24,25], and has a sensitivity and accuracy for the diagnosis of HCCA of $89 \%$ and $80-95 \%$, respectively[25,26].

Compared to ultrasound, CT can more intuitively and comprehensively depict the location and extent of involvement of the lesion, as well as invasion of the surrounding tissues and vascular system[27,28]. Typical CT manifestations of HCCA include dilatation of the intrahepatic bile ducts, irregular periductal thickening, intraluminal space occupancy, and the direct spread of tumors to the liver and adjacent blood vessels. CT-based diagnosis relies on the sudden transition between dilatation and non-dilatation of the bile duct[23]. MRI is a non-invasive and accurate method that provides detailed localization information and high soft-tissue resolution. When combined with MRCP, MRI can fully display the bile duct system and indicate bile duct involvement and secondary changes in the intrahepatic and extrahepatic bile ducts, while accurately assessing the size, classification, invasion extent, stenosis, blood vessel wall truncation, and tumor thrombus formation, with accuracy levels as high as 95\%[27,28].Positron emission tomography (PET)-CT with a

Page $4 / 10$ 
fluorodeoxyglucose (18-FDG) tracer detects the higher metabolism of cancer cells by measuring the tracer uptake in suspected lesions[23]. A suspected diagnosis can be excluded or confirmed by analyzing changes in PET-CT via focal concentrations and delayed imaging. This technique also enables the accurate location of adjacent tissue invasion, regional lymph nodes, and metastases to distant tissues and organs. Furthermore, PET-CT can improve the accuracy of staging and determination of distant metastasis before radical surgery, which facilitates the selection of an optimal treatment plan[24,25].

And endoscopic retrograde cholangiopancreatography (ERCP) provides information about the locations of tumors, morphology of bile ducts, and the relationship between tumors and bile ducts. This technique provides a clear image of the entire intrahepatic and extrahepatic bile ducts, which facilitates determinations of the locations and extent of lesions[25]. ERCP can also facilitate brush and cell biopsies to support a pathological diagnosis of HCCA[23]. Bile duct cell brush biopsy via ERCP or PTC is a common technique for obtaining a decisive diagnosis. Given the limited number of cells, however, this technique has a sensitivity of only $9-24 \%$, which increases to $35-44 \%$ with repeated brushing[25,30,31]. In summary, these imaging modalities each have unique advantages and are not interchangeable. Therefore, the use of these modalities in combination can help to differentiate benign from malignant lesions at the liver hilum for treatment purposes. However, a definite preoperative diagnosis of HCCA remains difficult.

Our study had some limitations of note. First, we did not perform a follow-up or survival analysis, but rather discussed only the choice of surgery. Second, the small number of patients and retrospective design may have reduced the reliability of our findings. Third, all of our patients resided in Northeast China, which may have led to substantial selection bias.

\section{Conclusion}

In conclusion, 11 out of 65 patients with presumed HCCA were misdiagnosed, despite improvements in the quality and number of imaging studies. No imaging feature or laboratory test finding reliably distinguished HCCA from benign lesions, which remains a difficult process. However, our findings confirm earlier reports in which a combination of painless jaundice and increased serum CA 19-9 and CEA levels tended to indicate HCCA in an otherwise undistinguishable case.

\section{List Of Abbreviations}

CEA:carcinoembryonic antigen:AFP:alpha fetoprotein; HCCA:hilar cholangiocarcinoma;ALT:alanine aminotransferase,AST:aspartate aminotransferase; ALKP:alkaline phosphatase;BILT:total bilirubin (BILT); BILD:indirect bilirubin.

\section{Declarations}

Ethics approval and consent to participate:the study was submitted to and approved by our institutional ethics committee.Consent was obtained from the patients.

Consent for publication:Not Applicable

Availability of data and material:Not applicable

Competing interests:The authors have no conflicts of interest to declare

Funding:This study was funded by the National Nature Science of China(81670580)(Literature research, design of the study) and Science \& Technology Support Project of Liaoning Province (201602831)(Grammatical improvement,collection, analysis, and interpretation of data).

Authors' contributions:

LW P:Literature research,Manuscript preparation

J K:Manuscript final version approval

All authors have read and approved the manuscript

Acknowledgements:Not applicable

\section{References}

Page 5/10 
1. Korosh K, Ur M, Wilson S R. Hilar biliary obstruction: preliminary results with Levovist-enhanced sonography. Ajr American Journal of Roentgenology, 2003, 180(3):687-693.

2. Klatskin G. Adenocarcinoma of the hepatic duct at its bifurcation within the porta hepatis: An unusual tumor with distinctive clinical and pathological features. American Journal of Medicine, 1965, 38(2):241-56.

3. Lang H , Kaiser G M, Zöpf T, et al. Surgical therapy of hilar cholangiocarcinoma. Der Chirurg, 2006, 77(4):325-34.

4. Thuluvath P J, Rai R, Venbrux A C, et al. Cholangiocarcinoma: a review. Gastroenterologist, 1997, 5(4):306-15.

5. Nakayama A, Imamura H, Shimada R, et al. Proximal bile duct stricture disguised as malignant neoplasm. Surgery, 1999, 125(5):51421.

6. Singh SS, Sharma R, Pal S, et al. Differentiation between benign and malignant hilar obstructions using laboratory and radiological investigations: A prospective study. HPB(Oxford) 2007; 9: 373-382.

7. Knoefel W T, Prenzel K L, Peiper M, et al. Klatskin tumors and Klatskin mimicking lesions of the biliary tree. European Journal of Surgical Oncology, 2003, 29(8):658-661.

8. Binkley CE, Eckhauser FE, Colletti LM. Unusual causes of benign biliary strictures with cholangiographic features of cholangiocarcinoma. Gastrointest Surg,2002; 6: 676-681.

9. Saxena A, Chua TC, Chu FC, Morris DL. Improved outcomes after aggressive surgical resection of hilar cholangiocarcinoma: a critical analysis of recurrence and survival. Am J Surg. 2011; 202:310-20.

10. DeOliveira ML, Cunningham SC, Cameron JL, Kamangar F, Winter JM, Lillemoe KD, Choti MA, Yeo CJ, Schulick RD. Cholangiocarcinoma: thirty-one-year experience with 564 patients at a single institution. Ann Surg. 2007; 245:755-62.

11. Kow AW, Wook CD, Song SC, Kim WS, Kim MJ, Park HJ, Heo JS, Choi SH. Role of caudate lobectomy in type III A and III B hilar cholangiocarcinoma: a 15-year experience in a tertiary institution. World J Surg. 2012; 36:1112-21.

12. Jaap Jacob Kloek, Otto Marinus van Delden, Deha Erdogan.Differentiation of malignant and benign proximal bile duct strictures: The diagnostic dilemma.World J Gastroenterol.2008:28; 14(32): 5032-5038.

13. Paritpokee $N$, Tangkijvanich $P$, Teerasaksilp $S$, et al. Fast liver alkaline phosphatase isoenzyme in diagnosis of malignant biliary obstruction. Journal of the Medical Association of Thailand, 1999, 82(12):1241-6.

14. Saluja SS, Sharma R, Pal S, Sahni P, Chattopadhyay TK. Differentiation between benign and malignant hilar obstructions using laboratory and radiological investigations: a prospective study. HPB (Oxford), 2007;9:373-82.

15. Larisa Vasilieva,Stefanos I. Papadhimitriou,Alexandra Alexopoulou.Clinical presentation, diagnosis, and survival in cholangiocarcinoma: A prospective study.Arab Journal of Gastroenterology,2016:17;181-184.

16. Juntermanns B, Radunz S, Heuer M, et al. Tumor markers as a diagnostic key for hilar cholangiocarcinoma. European Journal of Medical Research, 2010, 15(8):357-61.

17. Siqueira E, Schoen RE, Silverman W, Martin J, Rabinovitz M, Weissfeld JL, Abu-Elmaagd K, Madariaga JR, Slivka A. Detecting cholangiocarcinoma in patients with primary sclerosing cholangitis. Gastrointest Endosc 2002; 56: 40-47

18. Khan SA, Davidson BR, Goldin R, Pereira SP. Guidelines for the diagnosis and treatment of cholangiocarcinoma: consensus document. Gut.2002; 51 Suppl 6: VI1-VI9.

19. Hamano H, Kawa S, Horiuchi A, Unno H. High serum IgG4 concentrations in patients with sclerosing pancreatitis. N Engl J Med 2001; 344: $732-738$.

20. Ghazale A, Chari ST, Zhang L, Smyrk TC. Immunoglobulin G4- associated cholangitis: clinical profile and response to therapy. Gastroenterology 2008; 134: 706-715. 
21. Bamrungphon W, Prempracha N, Bunchu N, et al. A new mucin antibody/enzyme-linked lectin-sandwich assay of serum MUC5AC mucin for the diagnosis of cholangiocarcinoma. Cancer letters, 2007,247(2):301-308.

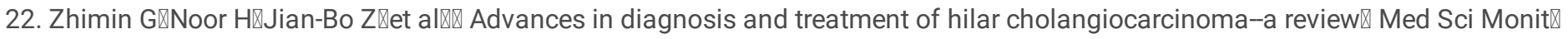
2013ه19: 648ه656

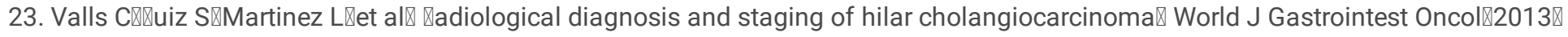
$5(7): 115 \rrbracket 126 \rrbracket$

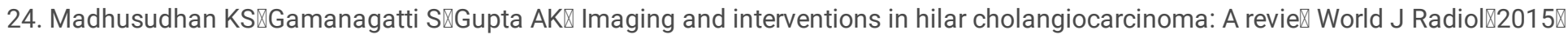
$7(2): 28 \llbracket 44 \rrbracket$

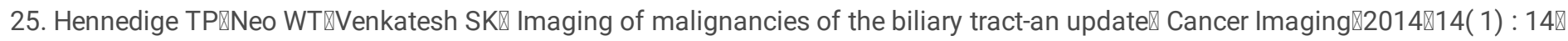

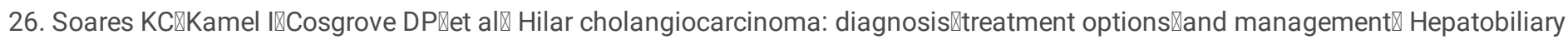
Surg Nutr $₫ 2014 \llbracket 3(1): 18 \rrbracket 34 \rrbracket$

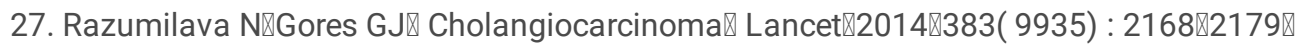

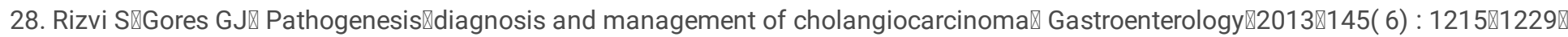

29. Sugita $\mathbb{\square}$ Magnetic resonance evaluations of biliary malignancy and condition at high-risk for biliary malignancy: Current status $\bigotimes$ World J Hepatolष2013囚5( 12) : 654囚665

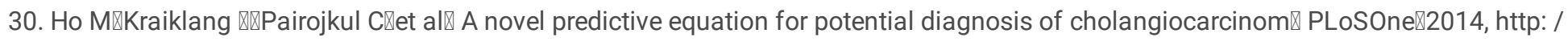
/journals $\rrbracket$ plos $₫$ org / plosone /article? id = 10. 1371 /journal $₫$ pone $₫ 0089337 \rrbracket$

31. Tamada KखUshio J『Sugano K囚 Endoscopic diagnosis of extrahepatic bile duct carcinoma: Advances and current limitations. World J

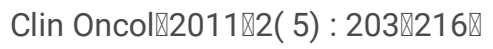

32. Hidalgo E, Asthana S, Nishio H, Wyatt J, Toogood GJ,Prasad KR, Lodge JP. Surgery for hilar cholangiocarcinoma:the Leeds experience. Eur J Surg Oncol. 2008;34:787-94.

33. Ercolani G, Zanello M, Grazi GL, Cescon M, Ravaioli M, Del Gaudio M, Vetrone G, Cucchetti A, Brandi G,Ramacciato G, Pinna AD. Changes in the surgical approach to hilar cholangiocarcinoma during an 18-year period in a Western single center. J Hepatobiliary Pancreat Sci. 2010;17:329-37

34. Li H, Qin Y, Cui Y, Chen H, Hao X, Li Q. Analysis of the surgical outcome and prognostic factors for hilar cholangiocarcinoma: a Chinese experience. Dig Surg. 2011;28:226-31.

35. Dumitrascu T, Chirita D, lonescu M, Popescu I. Resection for hilar cholangiocarcinoma: analysis of prognostic factors and the impact of systemic inflammation on long-term outcome. J Gastrointest Surg. 2013; 17:913-24

36. Croome KP, Rosen CB, Heimbach JK, Nagorney DM. Is Liver Transplantation Appropriate for Patients with Potentially Resectable De Novo Hilar Cholangiocarcinoma? J Am Coll Surg. 2015; 221:130-9.

\section{Tables}

[Table 1 could not be inserted due to technical limitations. It can be found in the supplemental files.]

Table 2. Comparison of the demographic, clinical, and laboratory characteristics between patients with hilar cholangiocarcinoma and those with benign bile strictures. 


\begin{tabular}{cccc}
\hline Group & Malignant & Benign & P value \\
\hline Sex $(\mathrm{M} / \mathrm{F})$ & $31 / 23$ & $3 / 8$ & 0.068 \\
Age $($ Years $)$ & $58(55,66)$ & $50(45,59)$ & 0.005 \\
\hline Time (days)* & $21(15,30)$ & $60(15,180)$ & 0.063 \\
\hline Jaundice & $46(85 \%)$ & $8(73 \%)$ & 0.210 \\
Abdominal discomfort & $31(57 \%)$ & $5(45 \%)$ & 0.693 \\
\hline Fever & $2(4 \%)$ & $1(9 \%)$ & 0.432 \\
\hline Weight loss & $9(17 \%)$ & $3(27 \%)$ & 0.689 \\
\hline CA19-9 $(\mathrm{U} / \mathrm{ml})$ & $259.8(82.63,577.28)$ & $37.98(11.63,151.6)$ & 0.002 \\
CEA $(\mathrm{ng} / \mathrm{ml})$ & $3.65(2.56,5.75)$ & $2.43(1.59,2.60)$ & 0.004 \\
\hline ALT $(\mathrm{U} / \mathrm{L})$ & $154(96,240)$ & $80(23,178)$ & 0.014 \\
\hline AST $(\mathrm{U} / \mathrm{L})$ & $111.5(61.5,162)$ & $80(28,131)$ & 0.077 \\
\hline ALKP $(\mathrm{U} / \mathrm{L})$ & $385.5(285.5,578.75)$ & $577(155,686)$ & 0.875 \\
\hline BILT $(\mu \mathrm{mol} / \mathrm{L})$ & $153.26(58.63,227.78)$ & $47.7(14.5,93.2)$ & 0.009 \\
\hline BILD $(\mu \mathrm{mol} / \mathrm{L})$ & $121.65(50.15,182.13)$ & $34.1(5.9,74.5)$ & 0.008 \\
\hline
\end{tabular}

Data are shown as medians and quartile ranges.

* Time from the appearance of symptoms to treatment.

Table 3. Imaging findings in the benign and malignant groups

\begin{tabular}{cccc}
\hline Imaging findings & Malignant & Benign & P value \\
\hline Hilar mass & $40(74 \%)$ & $8(73 \%)$ & 0.93 \\
Intrahepatic bile duct dilatation & $53(98 \%)$ & $10(91 \%)$ & 0.31 \\
\hline Bile duct wall thickening & $22(41 \%)$ & $6(55 \%)$ & 0.61 \\
\hline Lymph node enlargement & $15(28 \%)$ & $4(36 \%)$ & 0.84 \\
\hline Vascular invasion & $5(9 \%)$ & $0(0 \%)$ & 0.58 \\
\hline Gallbladder invasion & $9(17 \%)$ & $1(9 \%)$ & 0.86 \\
\hline
\end{tabular}

Table 4. Relevant evaluation data determined from the boundary points 


\begin{tabular}{lccllllll}
\hline $\begin{array}{l}\text { Diagnostic } \\
\text { boundary }\end{array}$ & Sensibility & Specificity & $\begin{array}{l}\text { Youden } \\
\text { index }\end{array}$ & $\begin{array}{l}\text { Positive } \\
\text { predictive } \\
\text { value }\end{array}$ & $\begin{array}{l}\text { Negative } \\
\text { predictive } \\
\text { value }\end{array}$ & $\begin{array}{l}\text { Positive } \\
\text { likelihood } \\
\text { ratio }\end{array}$ & $\begin{array}{l}\text { Negative } \\
\text { likelihood } \\
\text { ratio }\end{array}$ & \\
\hline $\begin{array}{l}\text { CA19-9 } \\
233.15\end{array}$ & $56 \%$ & $91 \%$ & 0.465 & 0.968 & 0.294 & 6.111 & 0.488 & 12.5 \\
$\mathrm{U} / \mathrm{ml}$ & & & & & & & & \\
\hline $\mathrm{CEA}$ & $61 \%$ & $90 \%$ & 0.585 & 0.949 & 0.514 & 6.85 & 0.35 & 19.57 \\
2.98 & & & & & & & & \\
$\mathrm{ng} / \mathrm{ml}$ & & & & & & & & \\
\hline
\end{tabular}

OR, odds ratio

\section{Figures}

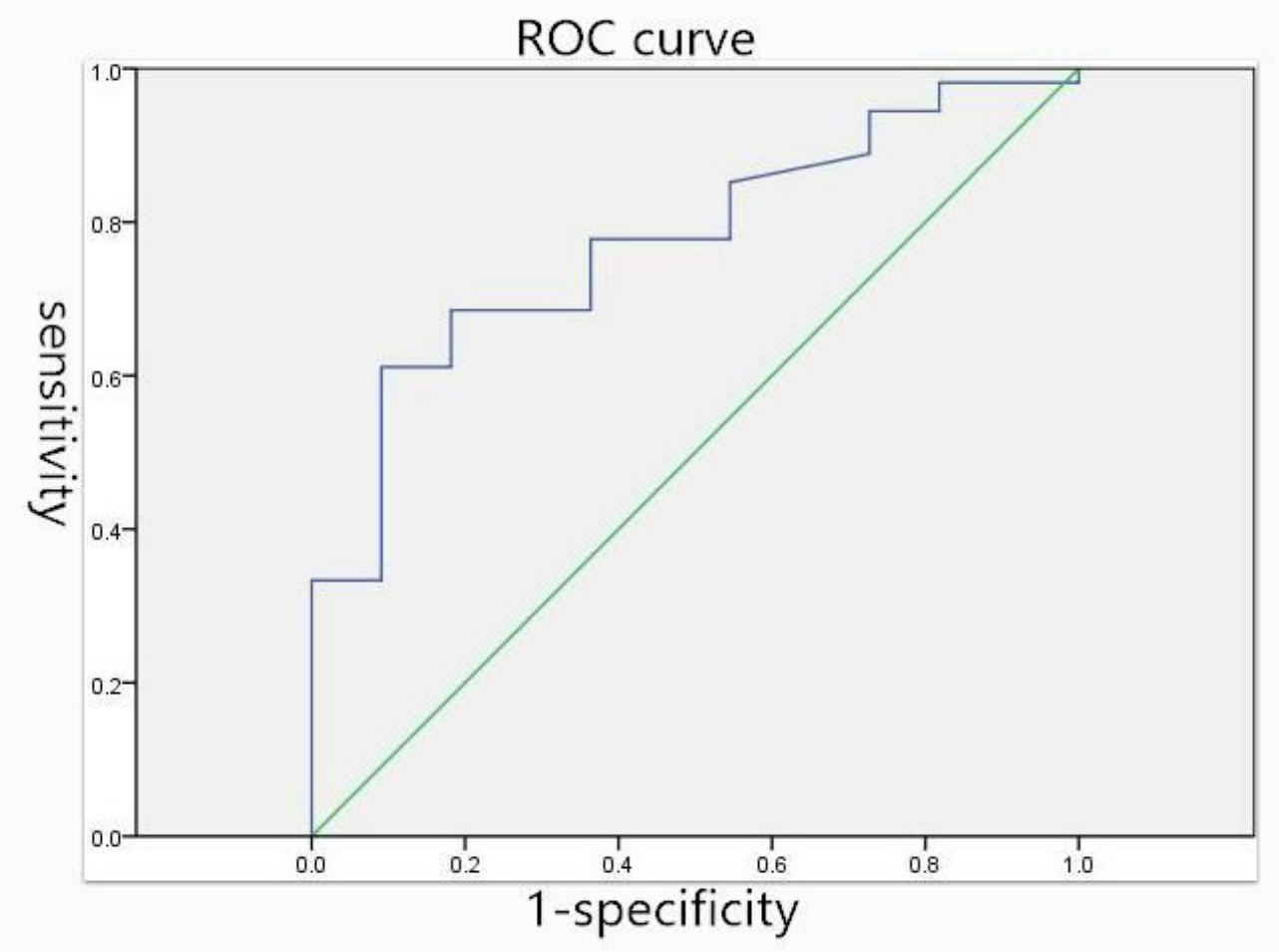

Figure 1

ROC curve analysis of CEA 


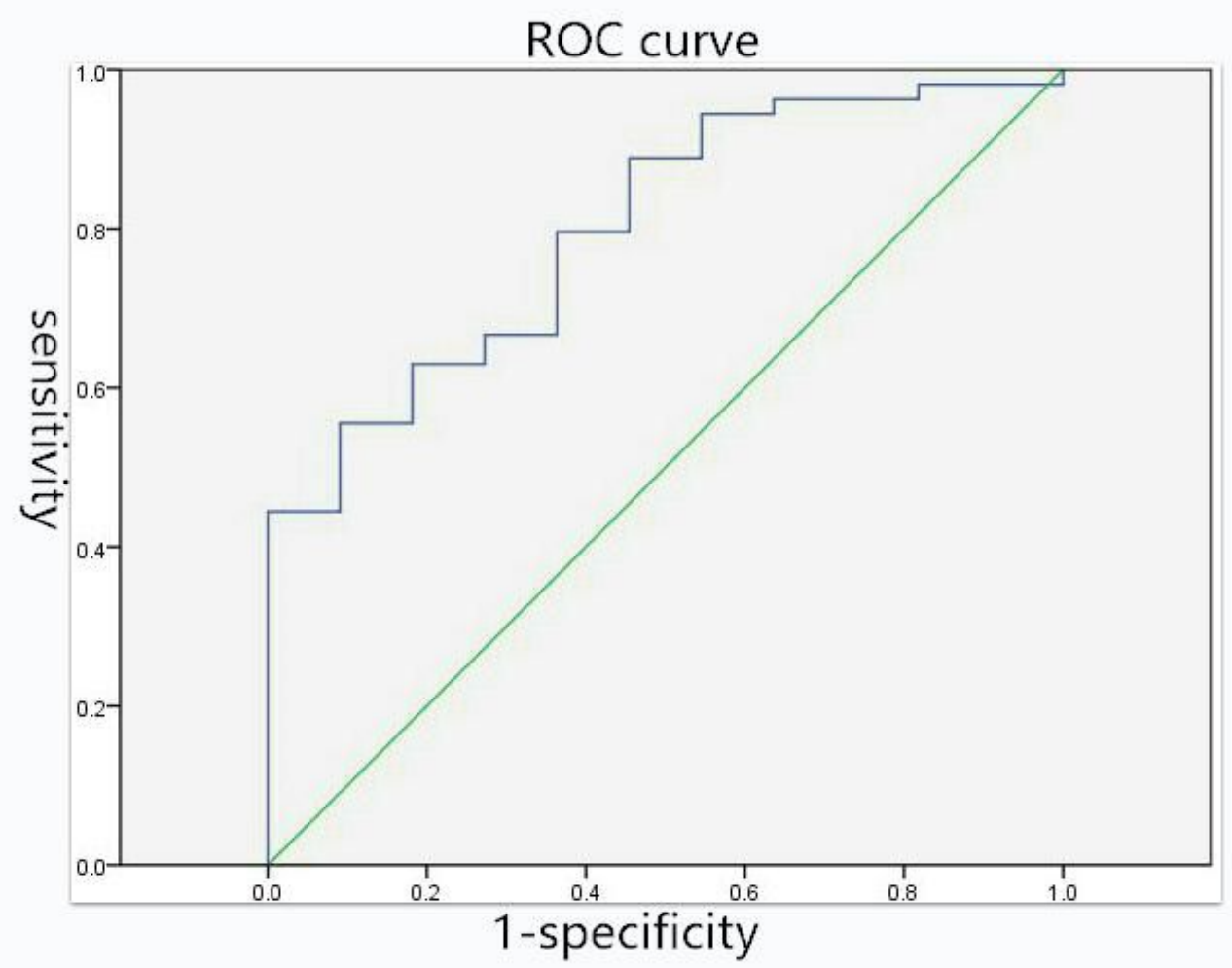

Figure 2

ROC curve analysis of CA19-9

\section{Supplementary Files}

This is a list of supplementary files associated with this preprint. Click to download.

- Table1.doc 